\title{
Longitudinal Analysis of Racial/Ethnic Trends in Quality Outcomes in Community Health Centers, 2009-2014
}

\author{
Megan B. Cole, $P h D, M P H^{1,2}$, Brad Wright, $P h D^{3}$, Ira B. Wilson, $M D^{2}$, Omar Galárraga, $P h D^{2}$, and \\ Amal N. Trivedi, MD, MPH
}

'Department of Health Law, Policy, \& Management, Boston University School of Public Health, Boston, MA, USA; '2Department of Health Services, Policy, \& Practice, Brown University School of Public Health, Boston, MA, USA; '3 Department of Health Management and Policy, University of lowa, lowa City, IA, USA.

BACKGROUND: To monitor progress towards eliminating health disparities, community health centers have reported on hypertension control, diabetes control, and birthweight by race and ethnicity since 2008 .

OBJECTIVE: To evaluate racial/ethnic time trends in quality outcomes in health centers and to assess both within- and between-center disparities in outcomes.

DESIGN AND SAMPLE: Using 2009-2014 data from all US health centers $(n=1047$ centers, serving 19.6 million patients/year), we evaluated racial/ethnic time trends in quality outcomes for health centers and assessed withinand between-center disparities.

MAIN MEASURES: Percentage of patients achieving control of blood pressure < 140/90 mmHg among hypertensive persons, control of glycosylated hemoglobin $\leq 9.0 \%$ among diabetic persons, and birthweight $\geq 2500 \mathrm{~g}$. All outcomes were reported by race/ethnicity.

KEY RESULTS: There was no evidence of improved outcomes among racial/ethnic subgroups from 2009 to 2014 , though electronic health record adoption, medical recognition, and insurance coverage rates increased substantially. Two exceptions were increased rates of normal birthweight for black patients $(87.0 \%$ to $88.8 \%$, or 0.3 percentage points/year, $p=0.02$ ) and decreased rates of diabetes control for white patients $(74.2 \%$ to $69.5 \%$, or -1.0 percentage points/year, $p<0.01)$. Within centers, the largest racial/ethnic disparities in 2009 were white/black disparities in hypertension control (8.7 percentage points, 95\% CI 7.4-10.1), white/black disparities in diabetes control (3.4 percentage points, 95\% CI 2.0-4.7), and white/ Hispanic disparities in diabetes control (4.4 percentage points, 95\% CI 2.8-6.0). All disparities remained statistically unchanged from 2009 to 2014 . White patients were more likely to be seen at a health center in the top performance quintile compared with black and Hispanic patients $(p<0.001)$.

CONCLUSIONS: Though quality outcomes in health centers continued to compare favorably to other care settings,

Electronic supplementary material The online version of this article (https://doi.org/10.1007/s11606-018-4305-1) contains supplementary material, which is available to authorized users.

Received July 6, 2017

Revised November 14, 2017

Accepted December 28, 2017

Published online February 16, 2018 we found no evidence of improved quality or reduced disparities in diabetes control, hypertension control, or birthweight from 2009 to 2014. Within- and betweencenter racial/ethnic disparities in quality were evident, and both should be targeted in future interventions.

KEYWORDS: community health centers; quality of care; health disparities;

hypertension; diabetes; birthweight.

J Gen Intern Med 33(6):906-13

DOI: $10.1007 /$ s11606-018-4305-1

(C) Society of General Internal Medicine 2018

\section{INTRODUCTION}

Community health centers are nonprofit organizations that provide comprehensive, quality primary care services to medically underserved communities, many of whom would otherwise be without access to care. ${ }^{1}$ Of the 22.9 million patients served by health centers in 2014 , the majority $(62 \%)$ were from racial/ethnic minority groups, while nearly all (92\%) had family incomes below $200 \%$ of the federal poverty level (FPL). Twenty-three percent of patients were best served in a language other than English, 28\% were uninsured, and $47 \%$ had Medicaid coverage. $^{2}$ Despite serving more vulnerable patient populations, studies suggest that the quality of care provided at health centers is generally similar to care provided in other settings. ${ }^{3,4,5,6}$ Research has also found that racial/ ethnic disparities in quality are much narrower within health centers than in other care settings, but disparities still exist. $^{4,7}$

Eliminating health and health care disparities for underserved populations is a key goal for health centers and many policymakers. To address disparities, health centers treat all patients without regard for ability to pay, must document and address the cultural and linguistic needs of their patient populations and have often participated in Health Resources and Services Administration (HRSA) Health Disparities Collaboratives to reduce disparities in chronic disease. $^{8,9}$ In 2008 , HRSA required all community health centers to report data on control of blood pressure in hypertension, control of blood sugar in diabetes, and birthweight by race/ethnicity to monitor 
progress towards eliminating disparities ${ }^{1} .{ }^{10}$ However, there is limited recent evidence about the extent to which disparities in quality outcomes have changed over time across health centers.

Our objective was to assess racial/ethnic trends in diabetes control, blood pressure control, and birthweight for health center patients from 2009 to 2014. We assessed both withincenter disparities, which measure differences between white and minority patients who receive care within the same center, and between-center disparities, which measure the extent to which racial/ethnic minority groups are concentrated in facilities where overall outcomes for both white and minority patients are worse. Understanding these two sources of disparities is important because they suggest distinct interventions to address inequalities among the health center population.

\section{METHODS}

Date Sources/Study Population. We used 2009-2014 Uniform Data System (UDS) data, which are collected annually by HRSA's Bureau of Primary Health Care. The UDS includes health center-level information on all Section 330 health center grantees $(N=1278)$. Each center reported data on quality measures, utilization of services, organizational features, and the characteristics of their treated patients. ${ }^{2}$ Due to the facilitylevel structure and compositional nature of the data, observations were analyzed and results are reported as percentages and not numbers. ${ }^{3}$

We excluded health centers located in US territories $(N=29)$; those where all sites were school-based, mobile, or seasonal $(\mathrm{N}=2)$; and those that were newly established or lost health center status during the study period $(N=200)$. Our final sample size was 1047 health centers, which served 17.8 million patients in 2009 and 21.2 million patients in 2014 (annual average: 19.6 million). For some analyses, we also excluded those with fewer than 20 patients in the outcome denominator; the size of these exclusions varied by measure.

Variables. The three outcome variables were percentage of patients achieving: (1) glycosylated hemoglobin (HbA1c) $\leq$ $9 \%$ among persons with diabetes, ${ }^{4}$ (2) blood pressure $<140 /$ $90 \mathrm{mmHg}$ among persons with hypertension, and (3) normal

\footnotetext{
${ }^{1}$ Select health centers reported on birthweight by race/ethnicity prior to 2008 , though reporting on diabetes control and hypertension control was required starting in 2008 .

${ }^{2}$ For a more detailed description of the Uniform Data System (UDS), including examples of prior literature using the UDS, please see Online Appendix.

${ }^{3}$ This is in contrast to individual-level analyses, where patient numerators and denominators may be reported. Though we analyze compositional data, the underlying patient denominators exceed 10,000 .

${ }^{4}$ While the reporting threshold used by HRSA is $\leq 9 \%$, which is reflective of the percentage of diabetic patients not exhibiting poor $\mathrm{HbA} 1 \mathrm{c}$ control, the recommended range for adequate control is $\leq 7 \%$, and thus the threshold used here is conservative.
}

birthweight ( $\geq 2500 \mathrm{~g}$ ) among patients giving birth. This represents all available outcome measures in the UDS. Measure definitions and data collection methods are described in the Online Appendix (eTable 1). All outcomes were reported annually by each center for non-Hispanic white, non-Hispanic black, and Hispanic patients. Outcomes were also reported for Asian and American Indian/Alaskan Native patients, but due to small sample sizes, results are reported in the Online Appendix only. For each health center, we calculated absolute disparities between non-Hispanic whites and each racial/ethnic group for each measure in each year. Covariates, all assessed at the health center level, included the following patient characteristics: percent male, percent age 65 or older, percent uninsured, percent with Medicaid coverage, percent with private coverage, percent with income below the FPL, percent homeless, and percent whose primary language was not English. We also included health center size (number of patients served), grant funding per patient, and an indicator for urban versus non-urban location.

TABLE 1 Health Center Characteristics, 2009 versus 2014

\begin{tabular}{|c|c|c|c|}
\hline Characteristic & 2009 & 2014 & $\begin{array}{l}p \text {-value of } \\
\text { difference }\end{array}$ \\
\hline Patients, no. & $17,819,154$ & $21,210,084$ & $<0.001$ \\
\hline \multicolumn{4}{|l|}{ Age, $\%$} \\
\hline $0-17^{*}$ & 28.6 & 27.2 & 0.013 \\
\hline $18-64$ & 63.9 & 64.0 & 0.864 \\
\hline $65+^{*}$ & 7.5 & 8.8 & $<0.001$ \\
\hline Male, \% & 42.5 & 42.9 & 0.207 \\
\hline \multicolumn{4}{|l|}{ Race/ethnicity, \% } \\
\hline Non-Hispanic White & 43.1 & 44.0 & 0.490 \\
\hline Non-Hispanic Black & 19.2 & 19.6 & 0.712 \\
\hline Hispanic & 25.3 & 25.7 & 0.709 \\
\hline Non-Hispanic Asian & 2.2 & 2.6 & 0.233 \\
\hline Non-Hispanic AIAN & 2.4 & 2.4 & 0.856 \\
\hline Other/unknown * & 7.8 & 5.7 & \\
\hline \multicolumn{4}{|l|}{ Insurance coverage, $\%$} \\
\hline Medicaid ${ }^{*}$ & 31.7 & 40.3 & $<0.001$ \\
\hline Medicare * & 8.1 & 9.9 & $<0.001$ \\
\hline Other Public * & 2.3 & 1.0 & $<0.001$ \\
\hline Private ${ }^{*}$ & 16.9 & 18.4 & 0.011 \\
\hline Uninsured* & 41.1 & $30.4^{\dagger}$ & $<0.001$ \\
\hline \multicolumn{4}{|l|}{ Income level, \% } \\
\hline Under $100 \%$ FPL & 68.7 & 67.9 & 0.341 \\
\hline Under $200 \%$ FPL & 91.9 & 91.3 & 0.260 \\
\hline $\begin{array}{l}\text { Primary language other } \\
\text { than English, \% }\end{array}$ & 17.8 & 16.5 & 0.166 \\
\hline Homeless, \% & 8.3 & 7.8 & 0.561 \\
\hline Veteran, $\%{ }^{*}$ & 1.5 & 1.9 & 0.005 \\
\hline Urban, $\%$ & 46.1 & 46.1 & NA \\
\hline Electronic Health & $40.0^{*}$ & 92.0 & $<0.001$ \\
\hline Record use, $\%^{*}$ & & & \\
\hline $\begin{array}{l}\text { Medical home } \\
\text { recognition, \%* }\end{array}$ & $35.2^{\S}$ & 65.4 & $<0.001$ \\
\hline
\end{tabular}

Statistics represent within-health center means, with each center weighted equally. Sample size is 1047 health centers, which includes 17.8 million patients in 2009 and 21.2 million patients in 2014. Characteristics for all years shown in Online Appendix C. "Difference between 2009 and 2014 is statistically significant, $p<0.01 .^{7}$ Large decline due 2014 insurance expansions; the 2013 uninsured rate was 37.4\%. Mean in 2011, when EHR was first reported in the UDS. ${ }^{\$}$ Mean in 2012, when medical home recognition was first reported in the $U D S$ 
Statistical Analysis. The unit of analysis was the health center. To assess the overall trend in outcomes for each racial/ethnic group, we used generalized estimating equations with exchangeable correlation structures to test whether performance rates changed over time for each group. We assessed the overall linear time trend by testing the significance of the year effect, where year is treated as a continuous variable in our model. We also estimated the marginal performance rates for each measure by year, where year is treated as an indicator variable in our model. In all analyses, we adjusted for the health center characteristics described above to account for compositional changes over time, weighted each observation by the population size (i.e., the outcome denominator), and used robust standard errors to account for weights. We clustered observations at the health center level to account for repeated measures.

To assess the overall trend in racial/ethnic disparities in outcomes within health centers, we used generalized estimating equations with exchangeable correlation structures to test whether the magnitude of disparity within health centers changed over time. We also estimated within-center disparities for each year (i.e., the marginal effect by year) using unadjusted measures. ${ }^{5}$ These within-center analyses were limited to health centers with at least 20 eligible white and 20 eligible minority patients in the measure denominator. ${ }^{11}$ All observations were clustered at the health center level to account for repeated measures.

To assess between-center disparities, we used the most recent year of data available at the time of the study (2014). We assigned health centers to quintiles based on race-standardized performance for each measure, where the performance of white non-Hispanic, black non-Hispanic, and Hispanic patients within each center was equally weighted. ${ }^{6}$ For example, a health center with diabetes control rates of $70 \%$ for whites, $60 \%$ for blacks, and $65 \%$ for Hispanics would have a race-standardized diabetes control rate of $(1 / 3 \times 0.7)+(1 / 3 \times 0.6)+(1 / 3 \times$ 0.65 ) or $65 \%$. Using denominator patient counts, we summed the total number of patients within each quintile for each race/ ethnicity and for each measure. We then estimated the odds of patients in each racial/ethnic group receiving care at a health center in the top $20 \%$ of all health centers versus a health center in the bottom $80 \%$ of all health centers. We calculated odds ratios between racial/ethnic groups using Pearson's chisquare test. Mean race-standardized performance rates by quintile are shown in the Online Appendix (eTable 11).

\footnotetext{
$\overline{{ }^{5} \text { Within-center }}$ disparities are unadjusted because health center characteristics are constant within a center for any given year for all two racial/ ethnic groups, as data are reported at the health center level. Further stratified data within a health center by race/ethnicity are unavailable. While aggregate outcomes within any racial/ethnic group should be adjusted for confounding factors, within a center, disparities themselves (or rather, differences in outcomes between two racial/ethnic groups) should not be adjusted, as this would suggest that in some types of health centers with larger proportions of different types of patients, larger disparities are permissible.

${ }^{6}$ See Online Appendix for example of prior research using a similar approach.
}

Sensitivity Analyses. In analyses of within-center disparities, we varied the minimum denominator specification (using alternative minimums of 1,5 , and 10 ). We also assessed time trends by race/ethnicity with and without weighting by population size. To account for the fact that EHR adoption was unavailable in the data from 2009 to 2010, we also repeated all primary analyses using only 2011-2014 data while adjusting for EHR adoption. To further explore potential biases introduced when a center begins using an EHR, we also examined trends in outcomes for centers switching from no EHR to an EHR compared with continuous EHR users and non-users. To account for the slightly skewed distribution of normal birthweight outcomes in the weighted analyses, we also repeat these analyses using generalized linear mixed models with a log-link. Finally, we stratified our analyses by US Census region to examine whether trends varied by region. All sensitivity analyses generated results that were largely consistent with our main findings and are reported in the Online Appendix.

All statistical analyses were performed using Stata v.14. Two-sided statistical tests with $p \leq 0.05$ were considered statistically significant.

\section{STUDY RESULTS}

Characteristics of Health Centers. The characteristics of our study population, stratified by the first and last year of the study period, are shown in Table 1. Between 2009 and 2014, we observed an 8.7 percentage point increase in the percent of health center patients with Medicaid coverage $(p<0.001)$ and a 10.7 percentage point decrease in the uninsured rate $(\mathrm{p}<0.001)$. The percent of centers using electronic health records (EHRs) increased from $67.0 \%$ in 2011 to $92.0 \%$ in 2014 , and the percent with patient-centered medical home (PCMH) recognition increased from $35.2 \%$ in 2012 to $65.4 \%$ in 2014. We detected increases in the percent of patients with Medicare coverage, with private coverage, with age over 65 , and who were veterans, but these changes were small in magnitude. Other characteristics were statistically unchanged.

Trends in Quality Outcomes by Race/Ethnicity. As shown in Fig. 1, from 2009 to 2014, unadjusted clinical performance across health centers remained relatively unchanged for each of the racial/ethnic groups. When testing the adjusted time trends using generalized estimating equations (Table 2), coefficients for most measures were small and insignificant $(p>0.05)$. One exception was rates of normal birthweight for black patients, which increased from $87.0 \%$ in 2009 to $88.8 \%$ in 2014 (adjusted annual change of 0.3 percentage points, $p=0.019$ ). A second exception was a statistically significant decline in diabetes control for white patients, from $74.2 \%$ in 2009 to $69.5 \%$ in 2014 (adjusted annual change of -1.0 percentage points, $p<0.001)$. Full estimates for all 

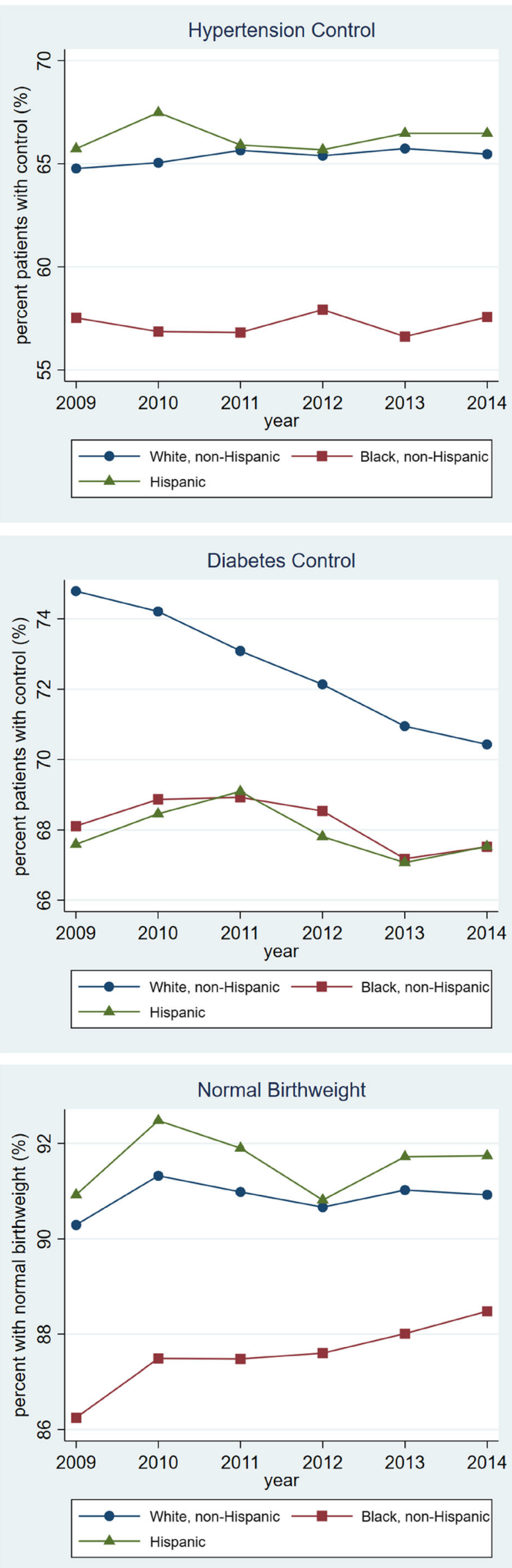

Fig. 1 Unadjusted health center patient outcomes by race/ethnicity, 2009-2014. Estimates shown represent the yearly, unadjusted marginal effects estimated from generalized estimating equations, using 2009-2014 Uniform Data System data. The rates shown represent health center population-level estimates or weighted means

measures by race/ethnicity are shown in the Online Appendix (eTable 3).

In stratified analyses by US Census region, results were mostly consistent across regions, with no region achieving consistent improvements in outcomes across all racial/ethnic groups (see Online Appendix eTable 9a-c).

Trends in Racial/Ethnic Disparities in Quality Outcomes Within Health Centers. As shown in the first column of Table 3, within health centers, statistically significant racial/ ethnic disparities in 2009 included a white/black disparity in hypertension control (8.73 percentage points, $p<0.001$ ), a white/black disparity in diabetes control (3.35 percentage points, $p<0.001$ ), a white/Hispanic disparity in diabetes control (4.40 percentage points, $p<0.001$ ), and a white/black disparity in normal birthweight $(2.58$ percentage points, $p<0.001)$.

The second and third columns of Table 3 show that racial/ ethnic disparities did not change over the 6-year study period. One exception was white/black disparities in controlled diabetes, which decreased at an average rate of 0.34 percentage points per year $(p=0.017)$. However, this was due to decreasing outcomes for whites over time $(p<0.001)$, as shown in Table 2. Though the rate of normal birthweight for blacks slightly increased over the study period, the white/black disparity remained statistically unchanged. Wide white/black disparities in hypertension control persisted, with a non-significant reduction of 0.04 percentage points per year $(p=0.724)$.

Racial/Ethnic Disparities Between Centers. As shown in Fig. 2, in 2014, black and Hispanic patients were consistently less likely than whites to receive care from health centers with the best race-standardized clinical outcomes. For hypertensive patients, the odds of receiving care at a health center in the top quintile of performance were lower for black $(\mathrm{OR}=0.65)$ and Hispanic $(\mathrm{OR}=0.43)$ patients compared with white, nonHispanic patients $(p<0.001)$. For diabetic patients, the odds of receiving care at a higher-performing health center were also lower for black $(\mathrm{OR}=0.84)$ and Hispanic $(\mathrm{OR}=0.70)$ patients compared with white, non-Hispanic patients $(p<0.001)$. Of pregnant women receiving care at health centers, black patients $(\mathrm{OR}=0.32)$ and Hispanic patients $(\mathrm{OR}=0.81)$ had lower odds of receiving care at a higher-performing health center compared with whites $(\mathrm{p}<0.001)$.

\section{DISCUSSION}

During our study period, health centers began systematically monitoring racial and ethnic disparities in blood pressure, 
TABLE 2 Adjusted Health Center Patient Outcomes by Race/Ethnicity, 2009-2014

\begin{tabular}{|c|c|c|c|c|c|c|c|}
\hline & \multirow[t]{2}{*}{$\mathbf{N}^{*}$} & \multicolumn{2}{|c|}{$\begin{array}{l}\text { Adjusted performance } \\
\text { in } 2009(95 \% \text { CI })\end{array}$} & \multicolumn{2}{|c|}{$\begin{array}{l}\text { Adjusted performance } \\
\text { in } 2014(95 \% \text { CI) }\end{array}$} & \multicolumn{2}{|c|}{$\begin{array}{l}\text { Mean adjusted annual } \\
\text { change from } 2009-2014 \\
(95 \% \text { CI })\end{array}$} \\
\hline & & \multicolumn{2}{|l|}{$\%$} & \multicolumn{2}{|l|}{$\%$} & \multicolumn{2}{|c|}{ Percentage points } \\
\hline \multicolumn{8}{|c|}{ Controlled hypertension } \\
\hline White & 1043 & 64.4 & $(63.2-65.6)$ & 64.4 & $(63.5-65.4)$ & 0.1 & $(-0.2$ to 0.3$)$ \\
\hline Black & 1018 & 58.8 & $(56.6-61.1)$ & 57.8 & $(56.4-59.1)$ & -0.2 & $(-0.6$ to 0.2$)$ \\
\hline Hispanic & 1023 & 65.7 & $(64.0-67.4)$ & 66.8 & $(65.3-68.2)$ & -0.1 & $(-0.4$ to 0.4$)$ \\
\hline \multicolumn{8}{|c|}{ Controlled diabetes } \\
\hline White & 1040 & 74.2 & $(73.2-75.3)$ & 69.5 & $(68.2-70.8)$ & -1.0 & $(-1.3$ to -0.7$)$ \\
\hline Black & 999 & 69.6 & $(68.2-71.1)$ & 69.4 & $(67.6-71.2)$ & -0.2 & $(-0.6$ to 0.2$)$ \\
\hline Hispanic & 1015 & 68.6 & $(67.1-70.1)$ & 68.4 & $(66.9-70.0)$ & -0.2 & $(-0.6$ to 0.2$)$ \\
\hline \multicolumn{8}{|c|}{ Normal birthweight } \\
\hline White & 908 & 90.8 & $(89.6-91.9)$ & 91.0 & $(90.2-91.9)$ & 0.0 & $(-0.2$ to 0.3$)$ \\
\hline Black & 762 & 87.0 & $(85.7-88.4)$ & 88.8 & $(87.8-89.8)$ & 0.3 & $(0.1$ to 0.6$)$ \\
\hline Hispanic & 812 & 91.2 & $(89.0-93.3)$ & 92.1 & $(90.2-93.9)$ & 0.0 & $(-0.3$ to 0.4$)$ \\
\hline
\end{tabular}

The adjusted performances reported in 2009 and 2014 represent the marginal, adjusted effects estimated from generalized estimating equations. The performance rates shown represent health center population-level estimates or weighted means. Performance rates are adjusted for confounding health center characteristics, including the percent of the health center patient population that was male, over age 65, uninsured, Medicaid insured, privately insured, under 100\% of FPL, homeless, whose primary language is not English, health center size (total number of patients served), grant funding per patient, and whether the center was located in an urban versus non-urban area. For unadjusted rates, see Online Appendix eTable 5 and Fig. $1 .{ }^{*} N$ indicates number of health centers. As shown, sample sizes vary by measure and racelethnicity and in 2014, represent 694,685-1,194,903 patients with hypertension, 330,750-527,793 patients with diabetes, and 46,477-131,304 patients giving birth.'The mean adjusted annual change represents the linear time trend from 2009 to 2014 or the "year" coefficient in our model

diabetes, and birth outcomes. Health centers also substantially increased their implementation of EHRs and patient-centered medical homes, and there were meaningful declines in uninsurance rates for health center patients. Despite these factors, clinical performance in blood pressure, diabetes, and birth outcome measures remained mostly unchanged from 2009 to 2014. The one important exception was diabetes control among white patients, which declined. Finally, we did not detect meaningful reductions in racial/ethnic disparities on the three outcome measures.

The lack of change mirrors trends observed in non-health center populations. The Agency for Healthcare Research and
Quality's (AHRQ's) National Disparity Report found that disparities in a broad set of quality measures have not narrowed over the last decade. ${ }^{12}$ For some indicators, disparities improved or were eliminated, but for others, disparities worsened over time. For diabetes control and hypertension control, research suggests that racial disparities have not significantly narrowed, ${ }^{13,14,15,16,17}$ though one study found that white/black disparities declined within the Medicare Advantage population in the western US Census region. ${ }^{16}$ Additionally, a study of the Veterans Affairs health care system population from 2000 to 2009 reported reductions in racial disparities in diabetes and hypertension control, though these

TABLE 3 Racial/Ethnic Disparities Within Health Centers, 2009-2014

\begin{tabular}{|c|c|c|c|c|c|c|c|}
\hline & \multirow[t]{2}{*}{$\overline{\mathbf{N}^{*}}$} & \multirow{2}{*}{$\begin{array}{l}\text { Absolute disparity in } 2009 \\
\text { (white-non-white) } \\
\text { (95\% CI) } \\
\text { Percentage points }\end{array}$} & \multicolumn{2}{|c|}{$\begin{array}{l}\text { Absolute disparity in } 2014 \\
\text { (white-non-white) } \\
(95 \% \mathrm{CI})\end{array}$} & \multicolumn{3}{|c|}{$\begin{array}{l}\text { Mean annual adjusted } \\
\text { change from } 2009 \text { to } 2014 \\
(95 \% \mathrm{CI})^{\dagger, *}\end{array}$} \\
\hline & & & Percentage points & & Percentage point & & \\
\hline \multicolumn{8}{|c|}{ Controlled hypertension } \\
\hline White/Black & 672 & 8.73 & (7.37 to 10.09 ) & 7.59 & (6.97 to 8.21$)$ & -0.04 & $(-0.28$ to 0.19$)$ \\
\hline White/Hispanic & 694 & 0.73 & $(-0.80$ to 2.24$)$ & -0.48 & $(-1.13$ to 0.18$)$ & -0.31 & $(-0.57$ to -0.05$)$ \\
\hline \multicolumn{8}{|l|}{ Controlled diabetes } \\
\hline White/Black & 577 & 3.35 & (2.02 to 4.68$)$ & 2.26 & (1.54 to 2.97$)$ & -0.34 & $(-0.62$ to -0.06$)$ \\
\hline White/Hispanic & 623 & 4.40 & (2.82 to 5.99$)$ & 2.48 & (1.71 to 3.25$)$ & -0.14 & $(-0.43$ to 0.14$)$ \\
\hline \multicolumn{8}{|l|}{ Normal birthweight } \\
\hline White/Black & 288 & 2.58 & (1.17 to 3.98$)$ & 1.95 & (0.98 to 2.93$)$ & -0.01 & $(-0.37$ to 0.19$)$ \\
\hline White/Hispanic & 384 & -2.49 & $(-4.69$ to -0.30$)$ & -1.39 & $(-2.55$ to -0.22$)$ & 0.20 & $(-0.12$ to 0.57$)$ \\
\hline
\end{tabular}

Estimates shown represent authors' calculations from generalized estimating equations. Disparity estimates in 2009 and 2014 represent the marginal, unweighted mean of absolute, within-center disparities between the two raciallethnic groups, where the mean rate for black or Hispanic patients is subtracted from the mean rate for non-Hispanic white patients. For all estimates, white patients are the referent group, where a positive disparity indicates a more favorable outcome for white patients and a negative disparity indicates a less favorable outcome for white patients. For all estimates, all centers are weighted equally; this excludes centers not contributing at least 20 patients to the denominator for each racial/ethnic group in the measure. "N represents number of centers contributing at least 20 patients to each denominator for the two racial/ethnic groups being compared. The number of patients represented varies by measure, racial/ethnic comparison, and year and is $>20,000$ for all measures. ${ }^{\dagger}$ The mean adjusted change represents the linear time trend measured by the model or the year coefficient. ${ }^{*}$ Adjusted for confounding health center characteristics, including the percent of the health center patient population that was male, over age 65, uninsured, Medicaid insured, privately insured, under 100\% of FPL, homeless, whose primary language is not English, health center size (total number of patients served), grant funding per patient, and whether the center was located in an urban versus non-urban area 


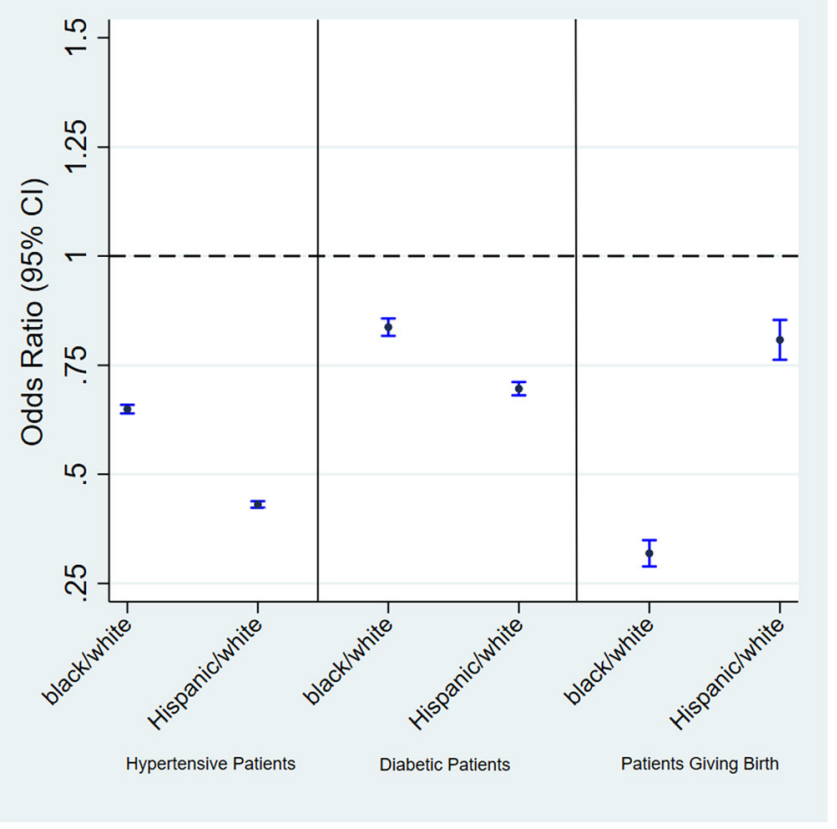

Fig. 2 Between-center disparities: Odds of minority versus white patients receiving care at a top-performing health center (2014). Estimates shown represent calculations from chi-square tests. The odds ratios (OR) represent the relative odds of a non-white patient receiving care at a health center in the top $20 \%$ (according to racestandardized quality rate) compared with the odds of a white, nonHispanic receiving care at a health center in the top $20 \%$. If an OR is $<1.0$, then non-white patients have lower odds of receiving care at a top performance health center compared with the white patient group. $p<0.001$ for all estimates

reductions were minimal. ${ }^{18}$ Research suggests that black/ white disparities for low birthweight slightly narrowed from 1980 to $2000,{ }^{19,20}$ but recent literature is limited.

The observed decline in diabetes control for white patients during the study period contrasts with research in broader patient populations suggesting flat or somewhat positive trends in diabetes control for whites during similar and earlier time periods. ${ }^{21,22}$ Diagnoses and incidence of diabetes also appear to be relatively flat during this time period, with small increases in prevalence and small decreases in incidence. ${ }^{23,24}$ One possible explanation for these results may be that risk factors for poor diabetes control, such as obesity, have changed differentially for white patients served by health centers compared with health center patients of other races/ethnicities and compared with white patients served in other settings. Testing this hypothesis would involve examining clinical, socioeconomic, behavioral, and environmental risk profiles in ways that our data do not permit.

Furthermore, lack of observed improvement in outcomes may indicate that some health centers, and the health care system more broadly, are not sufficiently resourced to target factors outside of the health care setting that contribute to outcomes, such as transportation, housing, food, and other social support. Organizational changes implemented during this time period, including widespread adoption of PCMH models and EHRs, largely do not address this gap. Although all community health centers provide some enabling services such as case management, transportation, medical benefit counseling, eligibility assistance for public insurance programs, and outreach, ${ }^{25,26}$ the scope of the services provided varies widely, and a center's ability to sufficiently provide these services may be constrained by staff time and resources. ${ }^{3,27,28}$ Lack of adequate access to specialty care and prescription drugs may also explain the difficulty in achieving improvements, particularly for the uninsured population.

Although we did not find evidence of improved quality outcomes or reduced disparities in outcomes, it is important to note that that the rates of achievement for controlled diabetes, controlled hypertension, and normal birthweight compare favorably to national averages, as found in a 2012 study by Shi et al. $^{3}$ This is further evident when comparing our results with national statistics. For instance, the National Health and $\mathrm{Nu}-$ trition Examination Survey reports hypertension control rates of $49.9 \%$ for non-Hispanic whites and $44.7 \%$ for nonHispanic blacks in 2011-2012 compared with our observed rates of $64.5 \%$ and $57.7 \%$ in the 2014 health center population. ${ }^{29}$ More so, racial/ethnic disparities within health centers are narrower than those observed in other care settings, ${ }^{7}$ and it may therefore be more difficult to close the remaining gap because of ceiling effects, particularly for birthweight.

Our results further indicate that both within and between center health disparities are contributing to the aggregate disparities observed across all health center patients. Within centers, although disparities are smaller than those observed in other populations, we detected substantial racial/ethnic gaps in quality outcomes, particularly between white and black hypertensive patients. Even within a center, where the providers, resources, and geographic setting are constant, disparities persist. This suggests the possibility of differential receipt of care within health centers for different racial/ethnic groups or differences in the complexity of the patients and the external barriers that they face. The latter suggests that racial/ethnic minority health center patients may disproportionately benefit from further expanding the role and capabilities of health centers in addressing health behaviors and social determinants of health.

Between-center results suggest that compared with white patients, black and Hispanic patients tend to receive care in centers where overall, race-standardized outcomes are lower. Even if disparities within every health center were eliminated, aggregate disparities in the health center population would persist. This suggests that efforts to further educate providers on equal treatment and promote cultural competency within health centers are not sufficient in addressing disparities, as racial/ethnic segregation into lower quality settings is evident. Prior literature suggests that a greater proportion of uninsured patients, fewer physicians, fewer enabling service providers, ${ }^{7}$ lower patient volume, and some degree of managed care penetration $^{3}$ may be associated with lower quality across all racial/ethnic groups. Additional research is needed to better understand characteristics associated with overall low quality of care at health centers, as focusing on these centers through 
targeted interventions and funding may disproportionally benefit racial/ethnic minority patients.

Our study has several limitations. First, we are unable to adjust outcome measures by patient-level characteristics, given that individual-level data are unavailable. However, these measures are not adjusted in the reporting done by HRSA, nor by other entities such as the National Committee for Quality Assurance that collect analogous measures, and clinical thresholds are set such that they should be achievable by all. We improve these estimates by adjusting for health center-level patient characteristics. Second, for our unweighted measures, by limiting analyses to centers reporting at least 20 patients in a denominator, we lose many health centers in our sample, yet still capture $70-97 \%$ of the patient population, depending on the measure. Third, we are unable to adjust for all health centerlevel confounders because of unmeasured characteristics, including health center-level staffing information and duration of operation, which are unavailable in our data, and EHR adoption and PCMH recognition, which are only reported in recent years. To further explore the role of EHR adoption, we repeat our analyses using limited 2011-2014 data while adjusting for EHR adoption and find that results are consist with our main findings. Fourth, we are unable to examine differential trends by insurance status, which may have differential distributions across racial/ethnic groups, as the UDS data are not further stratified by payer type. Fifth, while HRSA provides detailed guidance to health centers on measure reporting, measurement error certainly exists, though we have no reason to believe that the direction of error is non-random or differential over time. Finally, process measures by race/ethnicity that are related to the study outcomes are unavailable in our data.

\section{CONCLUSION}

Despite substantial investments and ongoing efforts to reduce disparities in health centers, performance on three key quality indicators assessing diabetes, hypertension, and pregnancy outcomes remained virtually unchanged from 2009 to 2014, with no evidence of reduced racial/ethnic disparities in outcomes. We found evidence of both within- and between-center disparities in quality outcomes, and both should be targeted in future interventions.

Acknowledgements: Funding for this work was provided by the Agency for Healthcare Research and Quality (1R36HSO2465201) and the Nora Kahn Piore Award.

Corresponding Author: Megan B. Cole, PhD, MPH; Department of Health Law, Policy, \& Management, Boston University School of Public Health, Boston, MA, USA (e-mail: mbcole@bu.edu).

\section{Compliance with Ethical Standards:}

Conflicts of Interest: We disclose that (1) Dr. Wilson helped Pfizer to organize and run a 1-day meeting on risk-benefit communication in 2016, for which he was paid as a consultant, and (2) Dr. Cole was employed part time by The Lewin Group - a subsidiary of Optum-from 2013 to 2017. All remaining authors declare that they do not have a conflict of interest.

\section{REFERENCES}

1. Health Resources and Services Administration (HRSA). What is a health center?. Rockville (MD): HRSA; 2015 Nov. Available at: http://bphc.hrsa. gov/about/what-is-a-health-center/index.html. Accessed Dec 11, 2017.

2. Health Resources and Services Administration (HRSA). 2014 Health Center Data. Rockville (MD): HRSA; 2015. Available at: https://bphc. hrsa.gov/uds/datacenter.aspx?year=2014Accessed Dec 11, 2017.

3. Shi L, Lebrun LA, Zhu J, Hayashi AS, Sharma R, Daly CA, et al. Clinical quality performance in US health centers. Health Serv Res. 2012; 47(6): 2225-2249.

4. Politzer R, Yoon J, Shi L, Hughes R, Regan J, Gaston M. Inequality in America: the contribution of health centers in reducing and eliminating disparities in access to care. Med Care Res Rev. 2001; 58(2):234-248.

5. Shi L, Tsai J, Higgins PC, Lebrun LA. Racial/ethnic and socioeconomic disparities in access to care and quality of care for US health center patients compared with non-health center patients. J Ambul Care Manage. 2009; 32(4):342-250.

6. Wright B, Potter A, Trivedi A. Federally Qualified Health Center use among dual eligibles: rates of hospitalizations and emergency department visits. Health Aff (Millwood). 2015; 34(7): 1147-1155.

7. Lebrun LA, Shi L, Zhu J, Sharma R, Sripipatana A, Hayashi AS, et al. Racial/ethnic differences in clinical quality performance among health centers. J Ambul Care Manage. 2013; 36(1): 24-34.

8. Health Resources and Services Administration (HRSA). Program Requirements. Rockville (MD): HRSA; 2016. Available at: http://www.bphc.hrsa. gov/programrequirements/index.html. Accessed Dec 11, 2017.

9. Hicks LS, O'Malley AJ, Lieu TA, Keegan T, McNeil BJ, Guadagnoli E, et al. Impact of Health Disparities Collaboratives on racial/ethnic and insurance disparities in US community health centers. Arch Intern Med. 2010; 170(3): 279-286.

10. Health Resources and Services Administration (HRSA). Efforts to expand and accelerate health center program quality improvement-report to Congress. Rockville (MD): HRSA; 2009. Available at: https://bphc.hrsa. gov/ftca/riskmanagement/healthcenterqualityimprovement.pdf. Accessed Dec 11, 2017.

11. Trivedi AN, Zaslavsky AM, Schneider EC, Ayanian JZ. Relationship between quality of care and racial disparities in Medicare health plans. JAMA. 2006; 296(16): 1998-2004.

12. Agency for Healthcare Research and Quality (AHRQ). National Healthcare Quality and Disparities Report. Rockville (MD): AHRQ; 2015.

13. Trivedi A, Zaslavsky AM, Schneider EC, Ayanian JZ. Trends in the quality of care and racial disparities in Medicare managed care. N Engl J Med. 2005; 353(7): 692-700.

14. McWilliams JM, Meara E, Zaslavsky AM, Ayanian JZ. Differences in control of cardiovascular disease and diabetes by race, ethnicity, and education: US trends from 1999 to 2006 and effects of Medicare coverage. Ann Intern Med. 2009; 150(8): 505-515.

15. Agency for Healthcare Research and Quality (AHRQ). National Healthcare Disparities Report, 2013: Chapter 2. Effectiveness of Care for Common Clinical Conditions. Rockville (MD): AHRQ: 2014

16. Egan B, Zhao Y, Axon RN. US trends in prevalence, awareness, treatment, and control of hypertension, 1988-2008. JAMA. 2010; 303(20):2043-2050.

17. Ayanian JZ, Landon BE, Newhouse JP, Zaslavsky AM. Racial and ethnic disparities among enrollees in Medicare Advantage plans. N Engl J Med. 2014; 371:2288-2297.

18. Trivedi A, Grebla R, Wright S, Washington D. Despite improved quality of care in the Veterans Affairs health system, racial disparity persists for important clinical outcomes. Health Aff (Millwood), 30(4): 707-715.

19. Branum AM, Schoendorf KC. Changing patterns of low birthweight and preterm birth in the United States, 1981-98. Pediatr Perinat Epidemiol. 2002; 16(1): 8-15.

20. Alexander GR, Slay M. Prematurity at birth: trends, racial disparities, and epidemiology. Ment Retard Dev D R. 2002; 8(4): 215-220.

21. Selvin E, Parrinello CM, Sacks DB, Coresh J. Trends in prevalence and control of diabetes in the United States, 1988-1994 and 1999-2010. Ann Inter Med. 2014; 160(8): 517-525. See Supplement. 
22. Wang Y, Katzmarzyl PT, Horsell R, Zhao W, Li W, Johnson J, et al. Racial disparities in the control status of cardiovascular risk factors in an underinsured population with type 2 diabetes. Diabet Med. 2014; 31(1): 1230-1236.

23. Centers for Disease Control and Prevention (CDC). Newly diagnosed diabetes and diagnosed diabetes: Age-adjusted rate per 1000 adults aged 18-76 years, 1996-2015. Atlanta (GA): CDC. Available at: https://gis. cdc.gov/grasp/diabetes/diabetesatlas.html. Accessed Dec 11, 2017.

24. Nichols GA, Schroeder EB, Karter AJ, Gregg EW, Desai J, Lawrence JM, et al. Trends in diabetes incidence among 7 million insured adults, 2006-2011. Am J Epidemiol. 2014; 181(1): 32-39.

25. Health Resources and Services Administration (HRSA). Health Center Program Terms and Definitions. Rockville (MD): HRSA; 2015. Available at: http://www.hrsa.gov/grants/apply/assistance/Buckets/definitions.pdf. Accessed Dec 11, 2017.
26. Health Resources and Services Administration (HRSA). Health Center Program Requirements. Rockville (MD): HRSA; 2014 February. Available at: http://bphc.hrsa.gov/about/requirements/hcpreqs.pdf. Accessed Dec 11, 2017.

27. Wells R, Punekar RS, Vasey J. Why do some health centers provide more enabling services than others? J Health Care Poor Underserved. 2009; 20(2): 507-523.

28. National Association of Community Health Centers (NACHC). Highlighting the role of enabling services at community health centers. Bethesda (MD): NACHC; 2010. Available at: http://nachc.org/wp-content/uploads/2015/06/EnablingServicesReport.pdf. Accessed Dec 11, 2017.

29. Centers for Disease Control and Prevention (CDC). National Center for Health Statistics, National Health and Nutrition Examination Survey. Atlanta (GA): CDC; 2013. Available at: https://nhqrnet.ahrq.gov/ inhqrdr/data/submit. Accessed Dec 11. 